\title{
ERRATUM
}

\section{Effectiveness of Respondent-Driven Sampling for Recruiting Drug Users in New York City: Findings from a Pilot Study}

Abu S. Abdul-Quader, Douglas D. Heckathorn, Courtney McKnight, Heidi Bramson, Chris Nemeth, Keith Sabin, Kathleen Gallagher, and Don C. Des Jarlais

Figure 3 was incorrectly printed in the original version of this article. The correct figure may be found below.
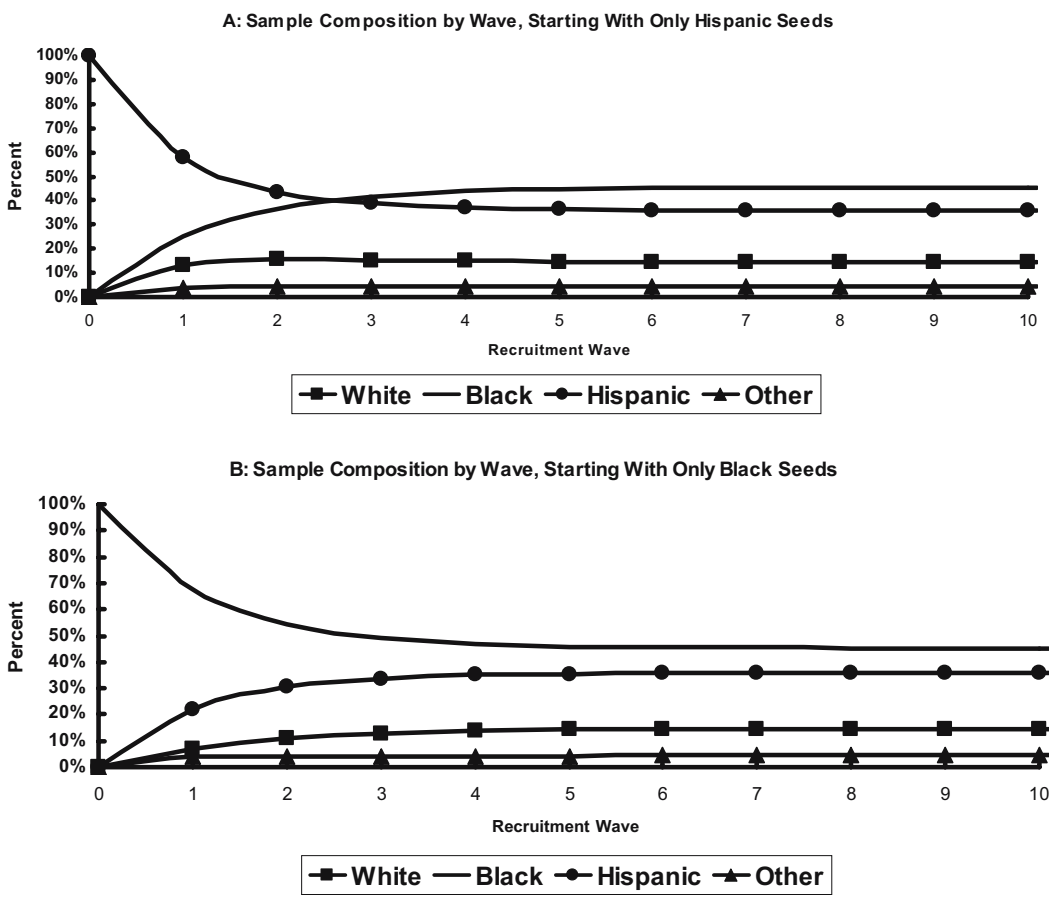

FIGURE 3. Sample composition stabilizes, reaching equilibrium independent of the choice of seeds. Recruitment by race/ethnicity, NYC drug users.

Abdul-Quader, Sabin, and Gallagher are with the Centers for Disease Control and Prevention, Atlanta, USA; Heckathorn is with the Department of Sociology, Cornell University, New York, USA; McKnight, Bramson, and Des Jarlais are with The Baron Edmond de Rothschild Chemical Dependency Institute, Beth Israel Medical Center, New York, USA; Nemeth is with the New York State Department of Health, Albany, USA.

Correspondence: Abu S. Abdul-Quader, Behavioral and Clinical Surveillance Branch, Division of HIV/AIDS Prevention-Surveillance and Epidemiology, National Center for HIV, STD and TB Prevention, Centers for Disease Control and Prevention, 1600 Clifton Road, MS E-46, Atlanta, GA 30333, USA. (E-mail: afa3@CDC.GOV)

The online version of the original article can be found at doi: 10.1007/s11524-006-9052-7. 